\title{
Asymmetric Flow in Symmetric Branched Structures
}

\author{
J. S. Andrade, Jr., ${ }^{1,2}$ A. M. Alencar, ${ }^{1,2,3}$ M.P. Almeida, ${ }^{1}$ J. Mendes Filho, ${ }^{1}$ S. V. Buldyrev,${ }^{2}$ S. Zapperi, ${ }^{2}$ \\ H. E. Stanley, ${ }^{2}$ and B. Suki ${ }^{3}$ \\ ${ }^{1}$ Departamento de Física, Universidade Federal do Ceará, 60451-970 Fortaleza, Ceará, Brazil \\ ${ }^{2}$ Center for Polymer Studies and Department of Physics, Boston University, Boston, Massachusetts 02215 \\ ${ }^{3}$ Department of Biomedical Engineering, Boston University, Boston, Massachusetts 02215
}

(Received 14 October 1997)

\begin{abstract}
We investigate the fluid flow through a cascade of bifurcations by direct simulation of the 2D Navier-Stokes equations. We show that, for a fully symmetric tree with $n$ generations $(n \geq 3)$, the flow distribution becomes significantly heterogeneous at an increased Reynolds number. We develop a binary tree model and find that the distribution of flow at the outlet branches can be described by a self-affine landscape, with a self-affine exponent $\alpha=0.9$ for the human lung. We suggest that the asymmetric flow occurring in symmetric branched structures may be important both for the morphogenesis of the bronchial tree, and for its functioning during inspiration. [S00319007(98)06724-6]
\end{abstract}

PACS numbers: 87.45.-k, 47.55.Mh

Fluid flow in branching geometries is related to many phenomena in physics, geology, and biology. Examples range from fluid flow through porous media $[1,2]$ to respiration [3] and blood circulation [4]. In particular, the mechanism of flow bifurcation plays a crucial role in the functioning of the respiratory and circulatory systems. The classical theoretical approach schematizes the flow with a set of equivalent linear impedances. When applied to steady or periodic flow through symmetric airway bifurcations, these models predict a perfectly homogeneous and synchronous flow distribution at the outlet branches [5]. However, the contribution of inertia on momentum transport can have a significant influence on the properties of flow through branched structures, as has been shown experimentally [6-10]. Despite recent numerical work [11], there has been no quantitative study in trees larger than three generations.

Here we simulate the quasisteady inspiration process in the bronchial tree. We consider a 2D symmetric cascade of rectangular channels with branching angles of $30^{\circ}$ between the axis of the parent and daughter generations, as well as realistic physiological dimensions from lung morphology to define the channel length and width (Fig. 1a) [5]. Because of the symmetry with respect to the axis of the first generation channel (trachea), the flow field in only half of the domain needs to be calculated. The fluid mechanics in the branched structure is based on the steady-state form of the Navier-Stokes and continuity equations for momentum and mass conservation. In all simulations, we consider air with density $\rho=1.225 \mathrm{~kg} \mathrm{~m}^{-3}$ and viscosity $\mu=1.7894 \times 10^{-5} \mathrm{~kg} \mathrm{~m}^{-1} \mathrm{~s}^{-1}$ flowing through the system at a constant flow rate with nonslip boundary conditions at the entire solid-fluid interface. In addition, we assume a uniform velocity profile at the inlet of the first generation channel, whereas at the outlets of the last generation branches, we impose a constant reference pressure [12]. The Reynolds number is $\operatorname{Re} \equiv \rho V d / \mu$, where $d$ is a characteristic length (the width of the first generation channel) and $V$ is the inlet velocity.

We solve the Navier-Stokes and continuity equations for the velocity and pressure fields by discretization, using the control volume finite-difference technique [13]. The creation of structured grids comprising quadrilateral elements is difficult due to the complex geometry of a binary tree structure. Hence, we use an unstructured mesh, based on triangular grid elements of a Delaunay network [14]. We find that a total of 17864 cells generates satisfactory results when compared with numerical meshes of smaller resolution. We then consider the integral form of the governing equations at each triangular element of the numerical grid to produce a set of coupled nonlinear algebraic equations, which we pseudolinearize and solve [13]. We achieve a converged solution when the sum of normalized momentum and mass residuals falls below $10^{-3}$ [15].

We perform fluid flow simulations in a five-generation tree for Re ranging from 150 to 4800 . This range corresponds to breathing flow rates that are physiologically relevant [3]. We find that the flow distribution throughout the airways is quite uniform at low Reynolds numbers (see Fig. 1a). In this situation the system displays linear behavior, as expected from the analogy between fluid flow in a cascade of branches and electrical transport in a network of ideal resistors [2]. At high Re, however, the nonlinear contribution from the inertial terms becomes relevant. As shown in Fig. 1b (for $\mathrm{Re}=4800$ ), inertial forces break the symmetry of the flow distribution down in the tree structure. Visual inspection reveals that the flow partitioning between any two daughter branches favors the branch which is aligned with their grandparent branch (branch located two generations above in the same cascade).

We investigate the development of this flow nonuniformity by gradually increasing Re and computing the flow rates at the outlets of the eight branches on the left side of the fifth generation. Figure 2 shows how these outlet 


\section{(1)}

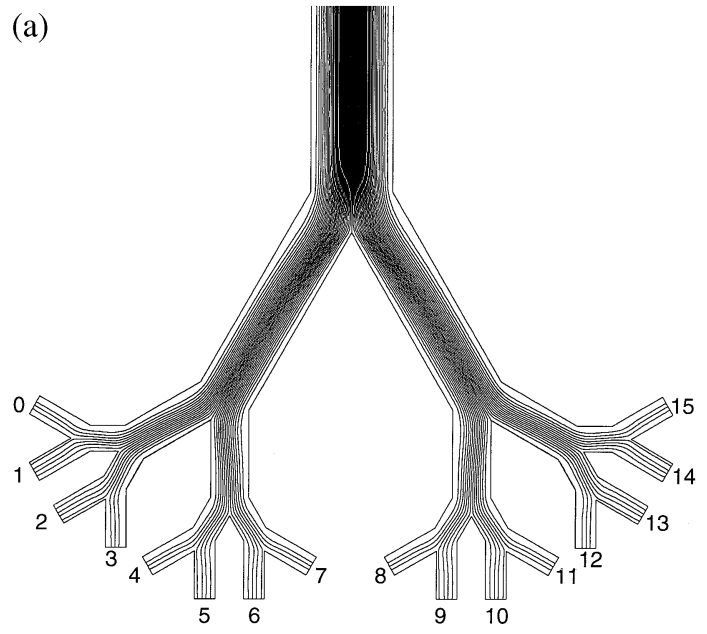

(b)

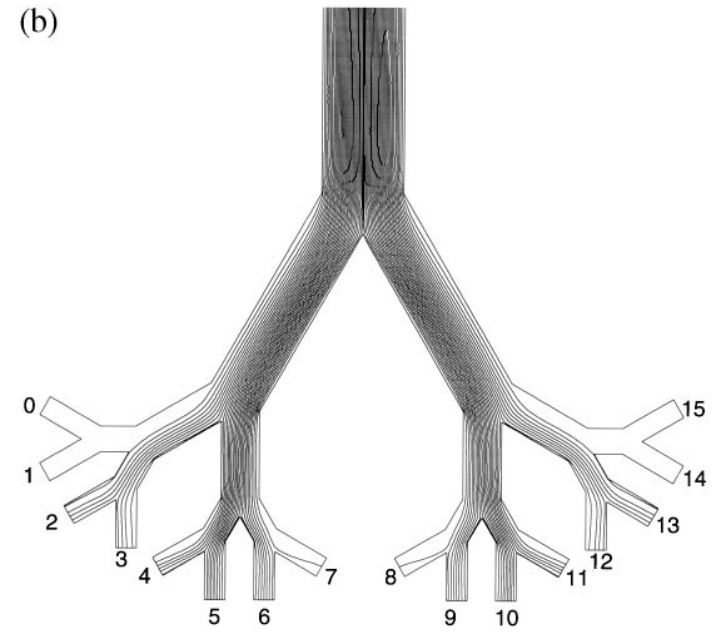

FIG. 1. (a) Contour plot of the stream function in a fivegeneration tree for low Reynolds number conditions $(\mathrm{Re}=$ 150). The tangents to the streamlines are parallel to the velocity vectors. For a given generation, the larger the number of streamlines in a branching element, the higher the flux. (b) Same as in (a), but for high Reynolds number conditions $(\operatorname{Re}=4800)$. The distribution of streamlines at the outlet branches is uniform at low Re, but highly nonuniform at high Re. The width of the first channel is $d=1.8 \mathrm{~cm}$ and the length is $l=12 \mathrm{~cm}$.

fluxes - normalized by the total flux penetrating the system-become more heterogeneous as Re increases. The normalized flow rates at outlets 5 and 10 are substantially increased by inertial effects.

Both Re and the branching angle $\theta$ influence the distribution of flow. To demonstrate this, we carried out simulations with a tree of only three generations (see Fig. 3). Figure 4 shows, for two different values of $\theta$, the dependence on $\operatorname{Re}$ of the ratio $G_{1} / G_{0}$ of the fluxes at the internal and external outlets of the third generation branches. For fixed branching angle and channel dimensions, both curves show a gradual increase of $G_{1} / G_{0}$ with Re. Furthermore, the value of $G_{1} / G_{0}$ is larger for $\theta=30^{\circ}$ than for $\theta=60^{\circ}$.

It would be interesting to study the effect of flow asymmetry on a large branching structure, but computational

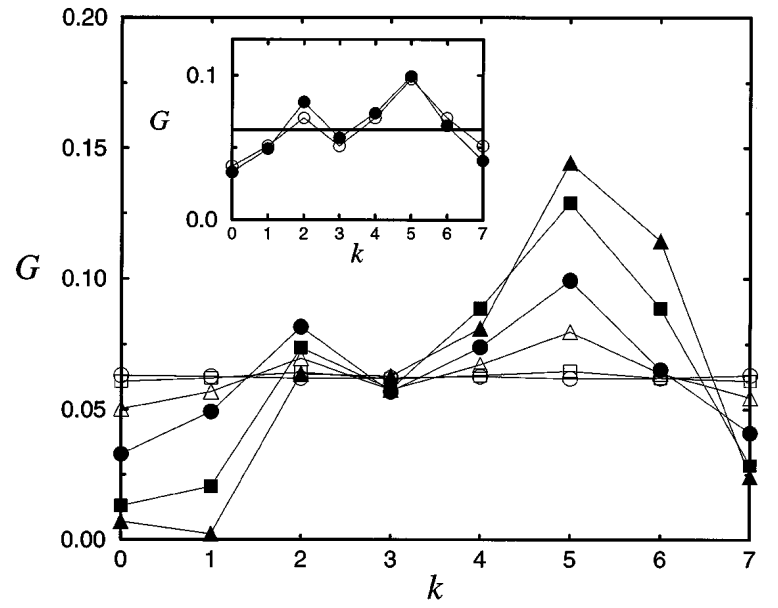

FIG. 2. Flux distribution at the outlet branches of a fivegeneration tree for $\operatorname{Re}=150$ (circle), 300 (square), 600 (triangle), 1200 (full circle), 2400 (full square), and 4800 (full triangle). The inset compares the fluid flow simulations $(\operatorname{Re}=1200$, circle) and the binary tree model $(p=0.58$, full circle).

limitations do not permit a direct solution of the NavierStokes equations. We therefore introduce a binary tree model to describe the role of inertia on fluid transport in a self-similar branching system. We assume that each airway forms an angle of $\pm \theta$, in degrees, with its parent. Also we assume that Re is sufficiently large for the flow partitioning to be approximately constant throughout the tree (e.g., the curve $\theta=60^{\circ}$ for $\operatorname{Re}>400$ in Fig. 4). Hence, the flow rates in any two daughter branches divide in a fixed proportion $p / q$, where $p+q=1$, and $p>q$. We model inertia by assigning the larger factor $p$ to the flow of the daughter branch which is aligned with its grandparent.

In order to treat the binary tree model analytically, we number all branches in generation $n$ from 0 to $2^{n-1}-1$. Thus, branch $k$ in generation $n-1$ bifurcates to branches $2 k$ and $2 k+1$ in generation $n$. Branch $2 k$ forms an angle $-\theta$ and branch $2 k+1$ forms angle $+\theta$ with their parent, so branch $2 k$ (even) is aligned with its grandparent if $k$ is odd, and branch $2 k+1$ (odd) is aligned with its grandparent if $k$ is even. Hence, a branch will be aligned with its grandparent if the two last digits in its binary representation are different. For a three-generation tree, the branches should have flows $q / 2, p / 2, p / 2$, and $q / 2$, corresponding to the binary codes $00,01,10$, and 11 , respectively. By induction, for a tree with $n$ generations, the flow in branch $k$ is

$$
G(n, k)=\frac{1}{2} p^{s(k)} q^{n-2-s(k),}
$$

where $s(k)$ is the number of "switches" from 1 to 0 and from 0 to 1 in the binary representation of $k$ with $n-1$ digits. In a seven-generation tree, branches 21 and 42 will have the maximum flow of $p^{5} / 2$ because their binary representations, 010101 and 101010, respectively, both have five switches. 

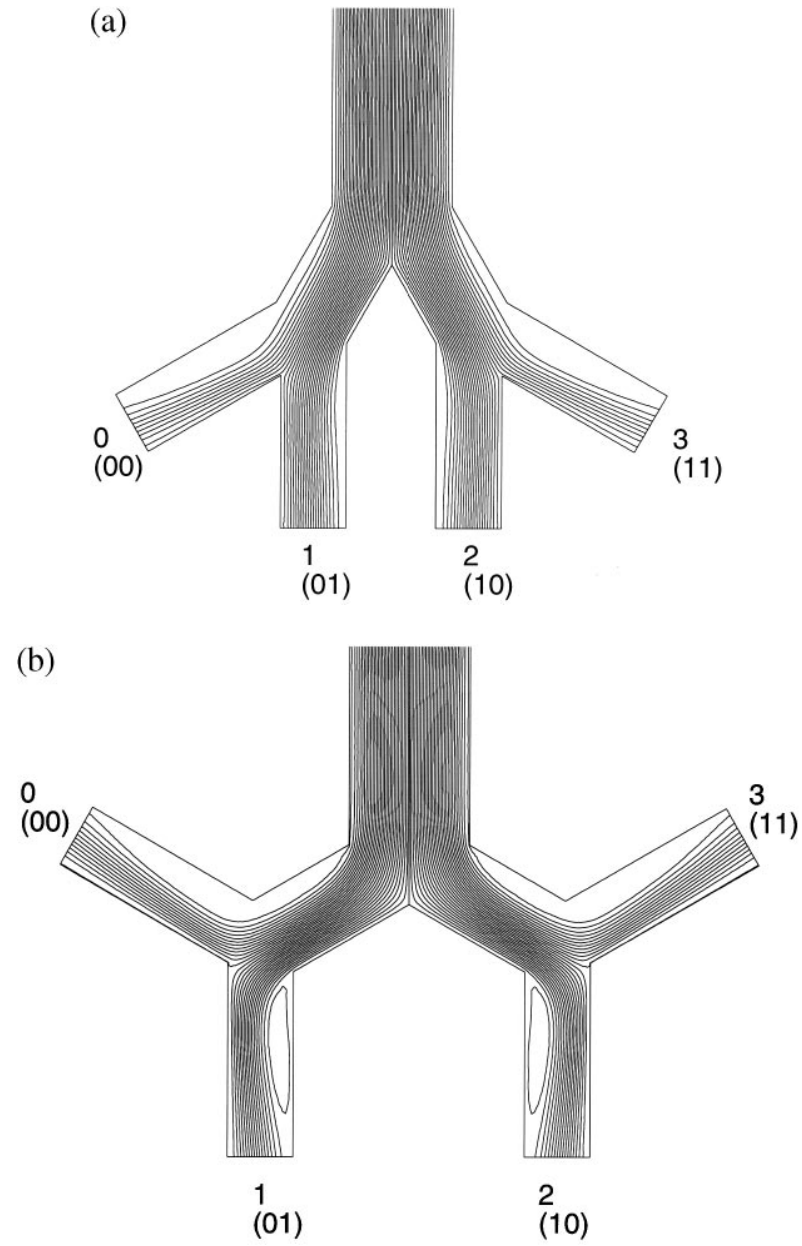

FIG. 3. (a) Contour plot of the stream function in a threegeneration tree with branching angle $\theta=30^{\circ}$ and $\operatorname{Re}=1200$. (b) Same as in (a) but for $\theta=60^{\circ}$. Note that the number of streamlines and thus the fluxes at outlets 1 and 2 are larger in (a) than in (b). Also shown is the binary representation of each outlet branch.

For the flow field calculations we need only to analyze the flow distribution through one-half, due to the symmetry of the tree. Thus, for generation $n$, we can normalize the flow $G$ in branch $k$ by the factor $p^{n-2} / 2$ and define

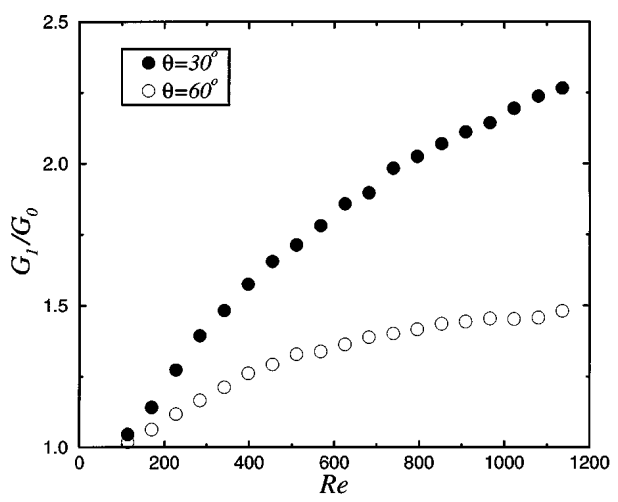

FIG. 4. Dependence of the flux ratio $G_{1} / G_{0}$ on the Reynolds number Re in three-generation trees for two different values of the branching angle $\theta$. the steplike flux function,

$$
g(x)=\left(\frac{q}{p}\right)^{n-2-s(k)} \text { for } \frac{k}{2^{n-2}} \leq x<\frac{(k+1)}{2^{n-2}},
$$

where $k=0,1, \ldots,\left(2^{n-2}-1\right)$. In this way, $g(x)$ is a well-defined right-continuous function for $n \geq 3$. For instance, the maximum flow $g^{\max }=1$ will be located at $x=2 / 3$ since its binary representation is $0.1010101 \ldots$ which has the maximum possible number of switches in the sequence [16]. In the limit $n \rightarrow \infty$, we find

$$
g(x)=\left(\frac{q}{p}\right)^{m} g\left(2^{m} x\right) \text { for } 0 \leq x<\frac{1}{2^{m}},
$$

which implies that the landscape (see Fig. 5) generated from the fluxes at the outlets of the ramified structure is self-affine, $g(x) \propto b^{-\alpha} g(b x)$, with an exponent [17]

$$
\alpha=\log (p / q) / \log 2 \text {. }
$$

Using the values of $p$ and $q$ reported in Ref. [18], we find $\alpha=0.9$ for the human lung and $\alpha=1.6$ for the more asymmetric dog lung.

The binary tree model provides insight on the effect of inertia on the flow distribution at the tree periphery. The self-affine structure of flow partitioning indicates a heterogeneous flow distribution; we expect that this feature does not depend on the approximations in the binary tree model, namely, constant angles and constant $p$ along the tree [19]. The exponent $\alpha$ is useful in quantifying the effect of asymmetry due to flow partitioning in large trees.

Concerning possible physiological implications, we note that our model predicts that, during inspiration, the amount of oxygen delivered to the periphery of the lung is very heterogeneous. The exact distribution depends on a weighted cumulative sum of the angles along which the air must travel from the top of the tree toward the alveoli where gas exchange occurs. On the other hand, the time constant inequalities along the airways are believed to be relatively small in the normal lung and hence the flow distribution should be uniform and primarily determined by the distribution of local compliances [20].

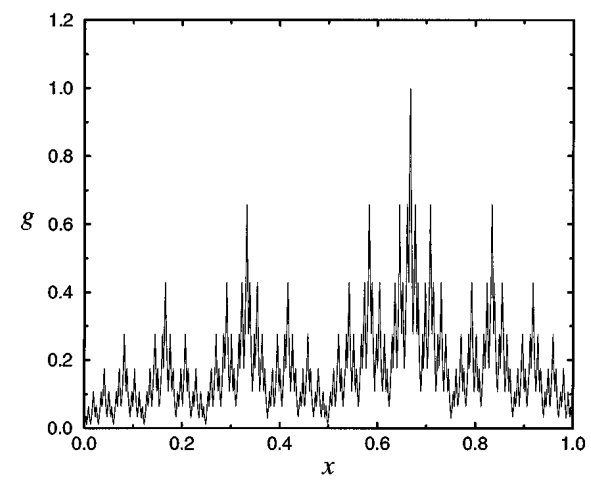

FIG. 5. Distribution of normalized fluxes $g(x)$ defined in Eq. (2) as a function of the normalized branch number $x$ at the outlets of an 11-generation tree with a partitioning factor $p=0.6$, calculated using the binary tree model. 
The expectation of a uniform lung ventilation seems therefore to be in contradiction with our results. We employed in our simulation a symmetric binary tree, whereas the geometrical structure of the tracheobronchial tree is highly asymmetric with respect to both angles and diameters of the daughter branches. It is known that the degree of asymmetry is larger in the shortest paths towards the periphery [21]. The shortest pathways occur in the directions of the lateral surface sides and the apex of the lung, where the angles are large and the flow is subject to many changes of direction. Moreover, in the Horsfield tree model, the diameters of the branches in the shortest pathways decrease rapidly, leading to smaller size subtrees [21]. Thus, we suggest that flow asymmetry due to inertial effects is compensated by structural asymmetry. Accordingly, the central airways (airways with a diameter larger than about $3 \mathrm{~mm}$ ) that receive the smaller flows serve correspondingly smaller alveolar regions - allowing for a homogeneous ventilation, as required for normal lung function.

We note that even though additional work with three dimensional tree models is needed to provide more quantitative predictions about the flow distribution during inspiration, our results still allow us to draw some important physiological conclusions. First, the fact that asymmetric flow distribution occurs even in a symmetric structure serves to justify previous lung flow models (see [18] and references therein) which must "build in" asymmetry in the flow partitioning in order to obtain results comparable with morphometric data. Second, our result could also have implications for lung morphogenesis. It was argued in Ref. [22] that the asymmetric structure of the lung is solely due to geometrical constraints, but our study suggests a possible different origin for this structure, since the asymmetry of the bronchial tree can be influenced by the fluid flow asymmetry combined with the requirement of homogeneous ventilation.

We thank NSF, CNPq, and FUNCAP for support.

[1] M. Sahimi, Applications of Percolation Theory (Taylor \& Francis, London, 1994); M. Sahimi, Rev. Mod. Phys. 65, 1393 (1993).

[2] P.M. Adler, Porous Media: Geometry and Transport (Butterworth-Heinemann, Stoneham, MA, 1992).

[3] R.H. Ingram, Jr., and T.J. Pedley, in Mechanics of Breathing, Handbook of Physiology: The Respiratory System (American Physiological Society, Bethesda, MD, 1986), Sec. 3, Vol. 3.

[4] D. MacDonald, Blood Flow in Arteries (Williams \& Wilkins, Baltimore, MD, 1974).

[5] T. J. Pedley, R.C. Schroter, and M.F. Sudlow, Respir. Physiol. 9, 387 (1970).

[6] B. Snyder, D. R. Dantzker, and M. J. Jaeger, J. Appl. Physiol. 51, 598 (1981).

[7] B. Snyder and M. J. Jaeger, J. Appl. Physiol. 54, 749 (1983).

[8] S. S. Slutsky, G. G. Berdine, and J. M. Drazen, J. Appl. Physiol. 49, 417 (1980).
[9] D. J. Isabey, J. Biomech. 15, 395 (1982).

[10] J. L. Allen, I. D. Frantz, and J. J. Fredberg, J. Clin. Invest. 76, 620 (1985).

[11] F. Wilquem and G. Degrez, J. Biomech. Eng. 119, 59 (1997).

[12] Experimental data obtained using the alveolar capsule technique indicate that at low frequencies (close to the breathing rate of $\approx 0.1 \mathrm{~Hz}$ ), the coefficient of variation of alveolar pressures in phase with lung volume is between 2\%-6\%; B. Suki et al., J. Appl. Physiol. 71, 69 (1991).

[13] S. V. Patankar, Numerical Heat Transfer and Fluid Flow (Hemisphere, Washington, DC, 1980). We use the FLUENT fluid dynamics analysis package (FLUENT Inc.) in this study.

[14] D. F. Watson, Comput. J. 24, 167 (1981).

[15] Residuals are computed for each equation by summing the imbalance in the equation for all cells in the domain. As an example, suppose a cell $i$ which is surrounded by a set of cells $j$. The normalized residual $R$ for a quantity $\phi$ is defined as

$$
R(\phi)=\sum_{i} \sum_{j}\left(A_{j} \phi_{j}-A_{i} \phi_{i}\right) / \sum_{i} A_{i},
$$

where the summation in $i$ is over all fluid cells in the lattice and the coefficients $A$ are the finite difference coefficients which combine convection and viscous terms through the control volume surrounding cell $i$. In general, a solution can be considered well converged if the normalized residuals are on the order of $10^{-3}$. For a given resolution of the numerical mesh, we usually perform an additional 100 iterations to test the stability of the converged solution. In addition, under-relaxation factors equal to 0.3 and 0.7 are used for the pressure and velocity variables, respectively.

[16] In the limit $n \rightarrow \infty, g(x)$ is equal to 0 for every $x$ except values of $x$ which are periodic (with period 01) in their binary representation. For all the other numbers $k-2-s(k) \rightarrow \infty$ as $n \rightarrow \infty$, and hence $g(x) \rightarrow 0$.

[17] If $2^{m} x$ has binary representation $0 . x_{1} x_{2} \cdots x_{k} 0101 \ldots$, then it is easy to show that $x$ is represented by $0.000 \cdots x_{1} x_{2} \cdots x_{k} 0101 \ldots$, which has $m$ switches less than the binary representation of $2^{m} x$. Hence, we obtain the prefactor $(q / p)^{m}$.

[18] H. Kitaoka and B. Suki, J. Appl. Physiol. 82, 968 (1997).

[19] In reality, $p$ may depend in a complex manner on the actual geometry. However, it is the mean value of $p$ that determines flow distribution in the periphery [18]. Using three-, four-, and five-generation trees, we find an average value of $p / q=1.45 \pm 0.03$ which gives $p \approx 0.59$. This value is consistent with $p=0.58$ used in the binary tree model to fit the flow data. The small variability $(\approx 6 \%$ of the mean) confirms our assumption that the $p / q$ ratio is approximately constant throughout the generations for sufficiently large Re values.

[20] J. Milic-Emili, The Lung: Scientific Foundations, edited by R.G. Crystal et al. (Raven Press, Ltd., New York, 1991).

[21] K. Horsfield, W. Kemp, and S. Phillips, J. Appl. Physiol. 52, 21 (1982).

[22] T.R. Nelson and D. K. Manchester, IEEE Trans. Med. Imaging 7, 321 (1988). 